\title{
AUTOMUTILAÇÃO EM ADOLESCENTES E ADULTOS JOVENS NA PANDEMIA POR COVID-19: O RELATO DE TRÊS CASOS
}

\author{
Janie Kelly Fernandes do Nascimento \\ Graduação em andamento em Medicina pela Universidade Iguaçu (UNIG), RJ, Brasil. E-mail: \\ janiekelly@hotmail.com \\ Nicolle dos Santos Moraes Nunes \\ Graduação em andamento em Medicina pela Universidade Iguaçu (UNIG), RJ, Brasil. E-mail: \\ nicolle.nunes_@hotmail.com \\ Marco Orsini \\ Médico, Pós-Doutorado em Neurologia pela Universidade Federal do \\ Rio de Janeiro, (UFRJ), RJ, Brasil. \\ Professor da Universidade de Medicina de Vassouras, RJ, Brasil. Professor Adjunto da \\ Universidade de Nova Iguaçu, (UNIG), RJ, Brasil. \\ orsinimarco@hotmail.com \\ Julia Fernandes Eigenheer Muhlbauer \\ Graduação em Medicina pela Universidade do Grande Rio (UNIGRANRIO), RJ, Brasil. \\ Especialização em andamento em Saúde da Família pela Universidade do Estado do \\ Rio de Janeiro (UERJ), RJ, Brasil. \\ juliafe24@yahoo.com.br
}

\section{RESUMO}

No final de 2019, o mundo acometido por uma grave patologia causada pelo vírus SARS-CoV2, denominada COVID-19. Acredita-se hoje que o isolamento social e a quarentena são as melhores estratégias para o controle da pandemia, pois, a partir deste binômio, é possível haver a redução da taxa de transmissão viral e, consequentemente, a diminuição nos casos de COVID-19. Entretanto, foi observado aumento substancial nas taxas de depressão, ansiedade, suicídio e automutilações não suicidas; principalmente em indivíduos com doença mental prévia. Conclui-se, então, que tais manifestações sejam reflexo do isolamento social, bem como do medo e incertezas gerados pela pandemia.

Palavras-chave: Automutilação. Adolescentes. Pandemia. Isolamento social.

\section{AUTOMUTILATION IN ADOLESCENTS AND YOUNG ADULTS IN PANDEMY BY COVID-19: THE REPORT OF THREE CASES}

\begin{abstract}
At the end of 2019 , the world was affected by a serious pathology caused by the SARS-CoV-2 virus, named COVID-19. Today, it is believed that social isolation and quarantine are the best strategies for controlling the pandemic, because, based on this binomial, it is possible to reduce the rate of viral transmission and, consequently, the decrease in the cases of COVID-
\end{abstract}


19. However, there has been a substantial increase in rates of depression, anxiety, suicide and non-suicidal self-harm; mainly in individuals with previous mental illness. It is concluded, then, that such manifestations are a reflection of social isolation, as well as the fear and uncertainties generated by the pandemic.

Keywords: Self-mutilation. Teens. Pandemic. Social isolation.

Recebido: $12 / 09 / 2020$.

Aceito: 14/10/2020. 


\section{INTRODUÇÃO}

No final de 2019, o mundo foi acometido por uma grave patologia causada pelo vírus SARS-CoV-2, denominada COVID-19. Moléstia esta que iniciou-se na China, na cidade de Wuhan, e que disseminou-se rapidamente para diversos continentes. A partir disso, em 11 de março de 2020, a Organização Mundial da Saúde (OMS) declarou status pandêmico (CUCINOTTA; VANELLI, 2020). Desde então, muito se tem pesquisado sobre o combate à propagação viral e sobre o tratamento da infecção, entretanto, até o momento, não há tratamento medicamentoso específico para a doença. Medidas gerais de isolamento foram bem estabelecidas a fim de se evitar sua disseminação. No entanto, tais atitudes carreiam consequências muitas vezes negligenciadas. (CUCINOTTA; VANELLI, 2020; DSOUZA et al., 2020).

Acredita-se hoje que o isolamento social e a quarentena são as melhores estratégias para o controle da pandemia, pois, a partir deste binômio, é possível haver a redução da taxa de transmissão viral e, consequentemente, a diminuição nos casos de COVID-19 (DSOUZA et al., 2020). No entanto, tais estratégias de saúde pública, apesar de trazerem os benefícios citados, possuem uma face desfavorável: a acentuação de distúrbios psiquiátricos, bem como comportamentos de caráter agressivo ou suicida na população. (HAWRYLUCK et al., 2004; BROOKS et al. 2020).

Muitos estudos acerca do impacto na qualidade de vida da população submetida ao isolamento obrigatório estão sendo realizados, havendo especial atenção para as repercussões na saúde mental. Foi observado um aumento substancial nas taxas de depressão, ansiedade, suicídio e automutilação não suicida; principalmente em indivíduos com doença mental prévia (CUCINOTTA; VANELLI, 2020; BROOKS et al. 2020). Conclui-se, então, que tais manifestações sejam reflexo do isolamento social, bem como do medo e incertezas gerados pela pandemia. (BROOKS et al. 2020; JEFSEN, 2020).

A automutilação consiste na destruição deliberada do tecido corporal próprio, socialmente não aceita e sem intenção suicida (IZADI-MAZIDI et al., 2019; NOCK; FAVAZZA, 2009). Os adolescentes representam a faixa etária mais vulnerável para esta prática (IZADIMAZIDI et al., 2019; NOCK; FAVAZZA, 2009), que pode resultar em graves consequências físicas, tais como lesões, infecções e outras complicações, incluindo óbito. (BRIERE; GIL, 1998; NOCK et al., 2006). 
A prática dessas autoagressões está também relacionada à promoção de sentimentos como vergonha e culpa, ao sofrimento emocional e a prejuízo do rendimento acadêmico (BRIERE; GIL, 1998; NOCK et al., 2006; TURNER; AUSTIN; CHAPMAN, 2014). É também considerada um potente preditor de tentativa de suicídio (ASARNOW, 2011; KLONSKY; MAY; GLENN, 2013). Em um estudo chinês, foram encontrados três fatores que podem ser enquadrados como causas para autolesão não suicida, são eles: regulação do afeto, influência social e evitação social (YOU; LIN; LEUNG, 2013). Ademais, fatores culturais, assim como as diferenças de gênero, também podem influenciar a prática. (IZADI-MAZIDI et al., 2019; GHOLAMREZAEI; DE STEFANO; HEATH, 2017; BENTLEY; NOCK; BARLOW, 2014).

O objetivo do presente artigo é expor, portanto, o relato de três casos de automutilação ocorridos durante a pandemia por SARS-Cov-2 e propor uma discussão sobre os motivos que regem tal desvio de comportamento.

\section{RELATO DOS CASOS}

\subsection{Caso 1}

FDP, 16 anos, sexo feminino, estudante. Paciente é conduzida ao atendimento médico por sua genitora devido a automutilações na região do antebraço e punho (Fig. 1). Afirma que os episódios foram vários, entretanto o último, na região da virilha, chamou atenção pela extensão e profundidade da lesão. Questionada sobre os motivos que a levaram à automutilação, a paciente disse não saber exatamente, mas que ao cortar-se sentia alívio da sensação de angústia que apresentava. Após distanciamento de sua genitora, a jovem também relatou uma sensação estranha de prazer: “Doutor, é uma espécie de dor boa que sinto, principalmente quando estou nervosa". Os cortes eram feitos por giletes elou barbeadores. Nega uso de drogas ilícitas e etilismo. Foi medicada e encaminhada para o serviço de psiquiatria. Atualmente em uso de escitalopram $10 \mathrm{mg} /$ dia; lamotrigina $12 / 12 \mathrm{~h}$ e divalproato de sódio à noite. Os episódios cessaram após 1 mês do início das medicações. 
Figura 1 - Automutilações na região do antebraço e do punho.

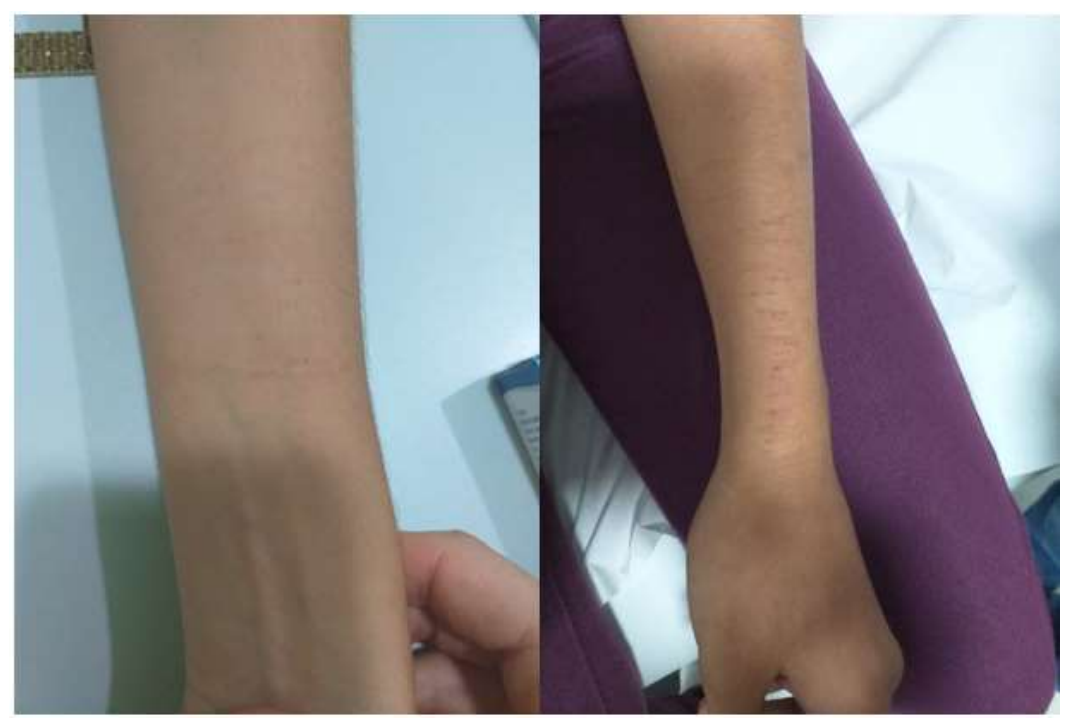

Fonte: Autor (2020).

\subsection{Caso 2}

JIN, 24 anos, sexo masculino, estudante de direito. Paciente relata que há aproximadamente seis meses vem apresentando sensações consideradas agradáveis após queimaduras com cigarros e cortes com gilete nas mãos e punho (Fig. 2). Relatou provocar as lesões de modo intencional, pois tinha em mente que se tratavam de cortes superficiais e não letais. Referiu ainda gostar de ver as marcas expostas em sua superfície corporal. Nega uso de drogas ilícitas e abuso de álcool. Nega história de doença psiquiátrica ou tratamento psiquiátrico prévio. Chama atenção a narrativa: "tenho total controle sobre o meu corpo sei a hora de parar". Iniciado tratamento com fluoxetina $40 \mathrm{mg} /$ dia; quetiapina $25 \mathrm{mg}$ de 12/12h e alprazolam $1 \mathrm{mg}$ à noite. O paciente apresentou substancial melhora do quadro, embora ocasionalmente ainda pratique automutilações com pequenos cortes. 
Figura 2 - Queimaduras com cigarros nos punhos.

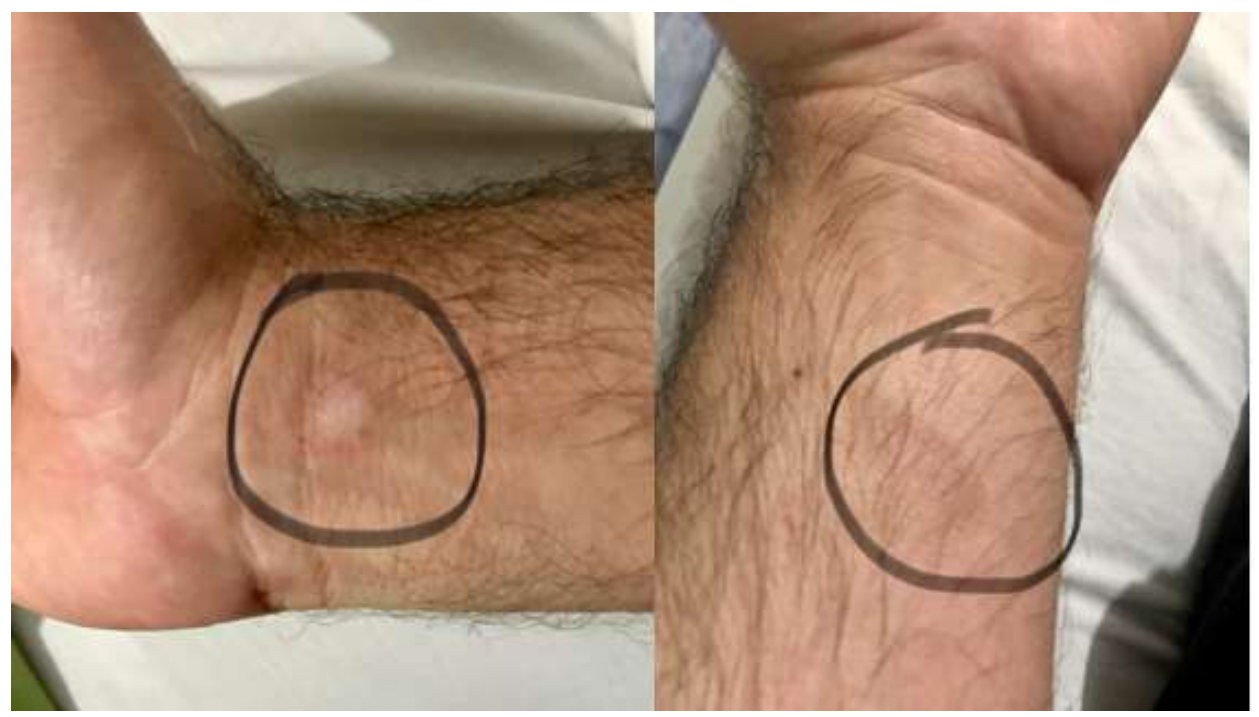

Fonte: Autor (2020).

\subsection{Caso 3}

ABS, 14 anos, sexo feminino, estudante do ensino médico. Paciente vem ao consultório médico acompanhada de seu genitor após sucessivos cortes na região do antebraço realizados com estiletes (Figura 3). O fato de algumas colegas de classe realizarem tais mutilações foi citado pela jovem como gatilho para início das autoagressões: "desde que minhas amigas começaram essas brincadeiras não consegui mais parar - sempre ao final do dia gosto de me cortar". Relata três episódios de ideação suicida, mas negou-se a detalhálas. Nega uso de drogas ilícitas e abuso de álcool. Referiu ainda sofrimento psíquico após separação e brigas entre os genitores; cobrança excessiva por bom desempenho escolar e término recente de um relacionamento. Atualmente em uso de escitalopram 10mg; lamotrigina 25mg de 12 em 12 horas e zolpidem $5 \mathrm{mg}$ à noite. Não houve remissão do quadro clínico após 30 dias do tratamento medicamentoso. 
Figura 3 - Cortes na região do antebraço realizados com estiletes.

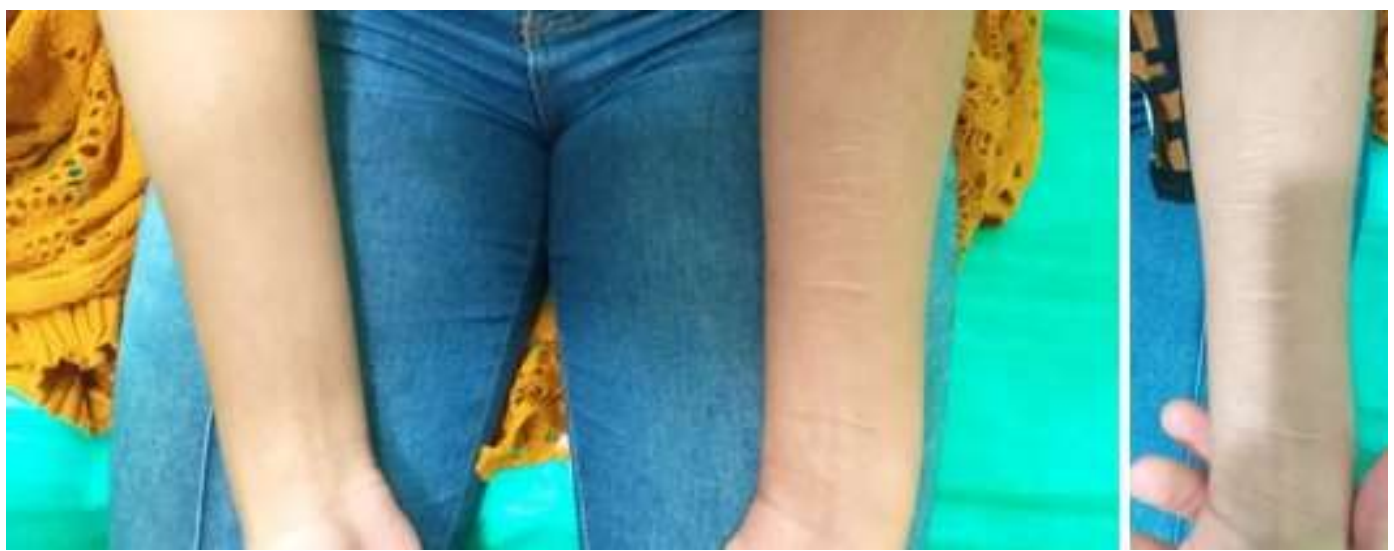

Fonte: Autor (2020).

\section{DISCUSSÃO}

Um artigo publicado recentemente pela Acta Psychiatrica Scandinavica observou como a pandemia provocada pelo Coronavírus poderia impactar tanto na saúde mental bem como nos cuidados psiquiátricos, e, além disso, estimou que os índices de suicídio e automutilações poderiam aumentar exponencialmente em decorrência da mesma. (BROOKS et al. 2020; JEFSEN, 2020; MADSEN; DINES; HIERONYMUS, 2020).

De fato, tal assertiva detinha certa veracidade, pois realmente constatou-se um aumento de tais comportamentos agressivos após a instalação da SARS-CoV-2 em Hong Kong (JEFSEN, 2020). Ademais, também pode ser constatado que pessoas com transtornos mentais prévios estão em situação de maior risco, haja vista que os devidos comportamentos estavam particularmente intensificados nesta população. (MADSEN; DINES; HIERONYMUS, 2020).

Diversas manifestações psicopatológicas têm sido associadas à pandemia. Entretanto, há duas que merecem maior destaque: a automutilação e o suicídio. Estas foram subdivididas em cinco esferas: pensamentos de auto-dano, auto-dano completo, desejo passivo de morte pela COVID-19, pensamentos suicidas e tentativas de realizar o suicídio (MADSEN; DINES; HIERONYMUS, 2020; QIN, 2011). Observou-se que os mais prevalentes eram as automutilações completas e o pensamento suicida; e que ambos possuíam maior prevalência na população feminina, com idade média de 29 a 37 anos. (QIN, 2011). 
É importante salientar que os desejos auto-agressivos não são gerados apenas pelo isolamento social, mas por um conjunto de crises sociais que a população mundial, em substancial parte, tem sofrido. Desemprego, pobreza e a falta de acesso à educação e saúde são situações que podem explicar a maior incidência de depressão e ansiedade, sendo, portanto, potenciais gatilhos para desencadear ou exacerbar transtornos psiquiátricos. (HAWRYLUCK et al., 2004; JEFSEN, 2020).

A população adulta, entretanto, não é a única a ser submetida a essas repercussões da pandemia na saúde mental. Crianças e adolescentes também experimentam desvios no comportamento, contudo, os motivos que os induzem a isso podem ser diferentes (DESLANDES; COUTINHO, 2020). Estudos recentes observaram que o consumo massivo de conteúdo digital, principalmente se relacionados à pandemia, contribuem para o desejo agressivo, especialmente à automutilação. (DESLANDES; COUTINHO, 2020; FERREIRA; DESLANDES, 2018). Tal prática, além de estar relacionada ao aumento dos índices de depressão, é também amplamente aceita e difundida no meio digital, por meio de brincadeiras e desafios online. (TORRES; VIVAS, 2012).

Devido a este cenário, a Organização Mundial da Saúde (OMS) desenvolveu cartilhas com o objetivo de orientar a população sobre questões psicossociais durante a pandemia da COVID-19. Dentre outras, é feita a recomendação de buscar apenas fontes de informações confiáveis e evitar ler notícias que possam gerar ansiedade. Além disso, é fortemente indicada a prática regular de exercícios físicos, bem como realizar atividades rotineiras com familiares e amigos de maneira segura e por meio de veículos digitais. (WORLD HEALTH ORGANIZATION, 2020).

Outra orientação de destaque é para os indivíduos estarem atentos ao possível surgimento de transtornos psicossociais advindos do isolamento social e do medo e incerteza causados pela moléstia. Além disso, que não hesitem em buscar ajuda profissional e em oferecer suporte às pessoas acometidas por tais manifestações, visto que a deterioração da saúde mental pode ter consequências sistêmicas no indivíduo em situação de vulnerabilidade psíquica e social. . (WORLD HEALTH ORGANIZATION, 2020; BANERJEE, 2020). 


\section{CONCLUSÃO}

Diversos relatos de casos e estudos já foram realizados a respeito das consequências psicossociais da pandemia da COVID-19. Tais estudos, em sua maioria, revelam que a COVID-19 está causando sofrimento psicológico à população devido às restrições impostas pelo isolamento social e ao medo da doença e suas consequências, sendo a automutilação uma das manifestações mais frequentes. Embora ainda se saiba pouco sobre a referida doença infecciosa, não há dúvidas que deve haver equilíbrio entre as estratégias de saúde implementadas para combatê-la e as políticas públicas para amenizar os impactos psicossociais decorrentes dela, visando o bem-estar físico e mental da sociedade como um todo.

\section{REFERÊNCIAS}

ASARNOW, J. R. et al. Suicide attempts and nonsuicidal self-Injury in the treatment of resistant depression in adolescents: findings from the TORDIA study. Journal of the American Academy of Child \& Adolescent Psychiatry, [S. I.], v. 50, n. 8, p. 772-781, 2011. DOI 10.1016/j.jaac.2011.04.003. Disponível em: https://jaacap.org/article/S08908567(11)00344-3/fulltext. Acesso em: 15 ago. 2020.

BANERJEE, D. The COVID-19 outbreak: crucial role the psychiatrists can play. Asian Journal of Psychiatry, [S. I.], v. 50, n. 102014, p. 1-2, 2020. DOI 10.1016/j.ajp.2020.102014. Disponível em: https://www.sciencedirect.com/science/article/abs/pii/S1876201820301258. Acesso em: 15 ago. 2020.

BENTLEY, K. H.; NOCK, M. K.; BARLOW, D. H. The four-function model of nonsuicidal selfinjury: key directions for future research. Clinical Psychological Science, [S. I.], v. 2, n. 5, p. 638-56, 2014. DOI 10.1177/2167702613514563. Disponível em: https://journals.sagepub.com/doi/10.1177/2167702613514563. Acesso em: 15 ago. 2020.

BRIERE, J.; GIL, E. Self-mutilation in clinical and general population samples: prevalence, correlates, and functions. American Journal of Orthopsychiatry, [S. I.], v. 68, n. 4, p. 609620, 1998. DOI 10.1037/h0080369. Disponível em: https://doi.apa.org/doiLanding?doi=10.1037\%2Fh0080369. Acesso em: 15 ago. 2020.

BROOKS, S. K. et al. The psychological impact of quarantine and how to reduce it: rapid review of the evidence. The Lancet, [S. I.], v. 395, n. 10227, p. 912-920, 2020. DOI: https://doi.org/10.1016/S0140-6736(20)30460-8. Disponível em: https://www.thelancet.com/journals/lancet/article/PIIS0140-6736(20)30460-8/fulltext. Acesso em: 15 ago. 2020. 
CUCINOTTA, D.; VANELLI, M. WHO declares COVID-19 a pandemic. Acta Biomed, [S. I.], v. 91, n. 1, p. 157-160, 2020. DOI 10.23750/abm.v91i1.9397. Disponível em:

https://www.mattioli1885journals.com/index.php/actabiomedica/article/view/9397. Acesso em: 15 ago. 2020.

DESLANDES, S. F.; COUTINHO, T. O uso intensivo da internet por crianças e adolescentes no contexto da Covid-19 e os riscos para violências autoinflingidas. Ciência \& Saúde Coletiva, [S. I.], v. 25, n. 202, p. 2479-2486, 2020. DOI 10.1590/1413-81232020256.1.11472020.

Disponível em: https://www.scielo.br/scielo.php?script=sci_arttext\&pid=S1413-

812320200067024793. Acesso em: 15 ago. 2020.

$1-$

2- $\quad$ DSOUZA, D. D. et al. Aggregated COVID-19 suicide incidences in India: fear of COVID19 infection is the prominent causative fator. Psychiatry Research, [S. I.], v. 290, n. 113145, p. 1-4, 2020. DOI 10.1016/j.psychres.2020.113145. Disponível em: https://www.sciencedirect.com/science/article/pii/S016517812031489X. Acesso em: 15 ago. 2020.

FERREIRA, T. R. S. C.; DESLANDES, S. F. Cyberbulling: conceituações, dinâmicas, personagens e implicações à saúde. Ciência \& Saúde Coletiva, [S. I.], v. 23, n. 10, p. 3369-3379, 2018. DOI 10.1590/1413-812320182310.13482018. Disponível em:

https://www.scielo.br/scielo.php?script=sci_arttext\&pid=S1413-81232018001003369.

Acesso em: 15 ago. 2020.

3-

GHOLAMREZAEI, M.; DE STEFANO, J.; HEATH, N. L. Nonsuicidal self-injury across cultures and ethnic and racial minorities: a review. International Journal of Psychology, [S. I.], v. 52, n. 4, p. 316-326, 2017. DOI 10.1002/ijop.12230. Disponível em:

https://onlinelibrary.wiley.com/doi/abs/10.1002/ijop.12230. Acesso em: 15 ago. 2020. 4-

HAWRYLUCK, L. et al. SARS control and psychological effects of quarantine, Toronto, Canada. Emerging Infectious Disease, [S. I.], v. 10, n. 7, p. 1-7, 2004. DOI 10.3201/eid1007.030703. Disponível em: https://www.ncbi.nlm.nih.gov/pmc/articles/PMC3323345/. Acesso em: 15 ago. 2020.

IZADI-MAZIDI, M. et al. Assessing the functions of non-suicidal self-injury: factor analysis of functional assessment of self-mutilation among adolescents. Iran J Psychiatry, [S. I.], v. 14, n. 3, p. 184-191, 2019.

JEFSEN, O. H. et al. COVID-19-related self-harm and suicidality among individuals with mental disorders. Acta Psychiatr Scand, [S. I.], v. 142, n. 2, p. 152-153, 2020. DOI 10.1111/acps.13214. Disponível em: https://onlinelibrary.wiley.com/doi/full/10.1111/acps.13214. Acesso em: 15 ago. 2020.

KLONSKY, E. D.; MAY, A. M.; GLENN, C. R. The relationship between nonsuicidal self-injury and attempted suicide: converging evidence from four samples. Journal Abnormal Psychology, [S. I.], v. 122, n. 1, p. 231-237, 2013. DOI 10.1037/a0030278. Disponível em: https://doi.apa.org/doiLanding?doi=10.1037\%2Fa0030278. Acesso em: 15 ago. 2020. 
MADSEN, M. M.; DINES, D.; HIERONYMUS, F. Optimizing psychiatric care during the COVID19 pandemic. Acta PsychiatrScand, [S. I.], v. 142, p. 70-71, 2020. DOI 10.1111/acps.13176. Disponível em: https://onlinelibrary.wiley.com/doi/full/10.1111/acps.13176. Acesso em: 15 ago. 2020.

NOCK, M. K. et al. Non-suicidal self-injury among adolescents: diagnostic correlates and relation to suicide attempts. Psychiatry Research, [S. I.], v. 144, n. 1, p. 65-72, 2006. DOI 10.1016/j.psychres.2006.05.010. Disponível em:

https://www.sciencedirect.com/science/article/abs/pii/S0165178106001351. Acesso em: 15 ago. 2020.

NOCK, M. K.; FAVAZZA, A. Nonsuicidal self-injury: definition and classification. In: NOCK, M. $K$. Understanding nonsuicidal self-injury: origins, assessment, and treatment. Washington, DC: American Psychological Association, 2009. p. 9-18.

QIN, P. The impact of psychiatric illness on suicide: differences by diagnosis of disorders and by sex and age of subjects. Journal of Psychiatric Research, [S. I.], v. 45, n. 11, p. 1445-1452, 2011. DOI 10.1016/j.jpsychires.2011.06.002. Disponível em: https://www.sciencedirect.com/science/article/abs/pii/S0022395611001105. Acesso em: 15 ago. 2020.

TORRES, F.; VIVAS, G. Comunicación electrónica y cyberbullying: Temas emergentes para la investigación e intervención socioeducativa. Psicología desde el Caribe, [S. I.], v. 29, n. 3, p. 707-730, 2012.

TURNER, B. J.; AUSTIN, S. B.; CHAPMAN, A. L. Treating nonsuicidal self-injury: a systematic review of psychological and pharmacological interventions. The Canadian Journal of Psychiatry, [S. I.], v. 59, n. 11, p. 576-585, 2014. DOI 10.1177/070674371405901103. Disponível em: https://journals.sagepub.com/doi/10.1177/070674371405901103. Acesso em: 15 ago. 2020.

WORLD HEALTH ORGANIZATION. Mental health and psychosocial considerations during the COVID-19 outbreak. [Genebra]: WHO, 2020. Disponível em: https://apps.who.int/iris/bitstream/handle/10665/331490/WHO-2019-nCoV-MentalHealth2020.1-eng.pdf. Acesso em: 15 ago. 2020.

YOU, J.; LIN, M. P.; LEUNG, F. Functions of nonsuicidal self-injury among Chinese community adolescents. Journal of Adolescence, [S. I.], v. 36, n. 4, p. 737-745, 2013. DOI 10.1016/j.adolescence.2013.05.007. Disponível em: https://www.sciencedirect.com/science/article/abs/pii/S0140197113000730. Acesso em: 15 ago. 2020. 